\title{
Abstract
}

\section{A Comparison of the Economic Status of Working-Age Persons with Visual Impairments and Those of Other Groups}

\author{
Andrew J. Houtenville
}

This article compares the economic status of working-age individuals with visual impairments with those with nonvisual impairments. It shows that the employment rates and mean household incomes were lower and the receipt of Social Security Disability Insurance was higher among those who were blind in both eyes than among those with less-severe visual impairments and those who were deaf in both ears.

Published in:

Journal of Visual Impairment and Blindness. Volume 97, Number 03, March 2003. Pages: 133-148.

For full text see publisher's website: http://www.afb.org/jvib.asp 